\section{Sociodemographic Predictors of SARS-CoV-2 Infection in Obstetric Patients, Georgia, USA}

\author{
Naima T. Joseph, Kaitlyn K. Stanhope, \\ Martina L. Badell, John P. Horton, Sheree L. Boulet, \\ Denise J. Jamieson
}

Author affiliation: Emory University School of Medicine, Atlanta, Georgia, USA

DOI: https://doi.org/10.3201/eid2611.203091

We conducted a cohort study to determine sociodemographic risk factors for severe acute respiratory syndrome coronavirus 2 infection among obstetric patients in 2 urban hospitals in Atlanta, Georgia, USA. Prevalence of infection was highest among women who were Hispanic, were uninsured, or lived in high-density neighborhoods.

$\mathrm{D}$ ata from New York, New York, USA, have highlighted the disproportionate burden of coronavirus disease (COVID-19) in minority and low socioeconomic status communities in the United States (1), yet data are lacking from southeastern states, which are home to a large share of the nation's racial and ethnic minority populations $(2,3)$. The information is imperative in states like Georgia, which has average of 3,0004,000 new cases daily and an urgent need for improved public health mitigation and containment strategies (4). The objective of this study was to determine associated sociodemographic and neighborhood risk factors for infection in an obstetric cohort undergoing testing for severe acute respiratory syndrome coronavirus 2 (SARS-CoV-2) in 2 urban hospitals in Atlanta, Georgia. Emory University School of Medicine Institutional Review Board and Grady Memorial Hospital Research Oversight Committee approved the study.

Universal testing for SARS-CoV-2 was initiated on all parturients admitted to labor and delivery at Grady Memorial Hospital, a safety-net hospital in Atlanta, starting on April 20, 2020, and at Emory University Hospital Midtown, an academic hospital in Atlanta, starting on April 24. Both hospitals serve a diverse, predominantly minority population; the safety-net hospital captures a larger proportion of uninsured and Medicaid patients (5). The safety-net hospital performs $\approx 2,500$ and the academic hospital $\approx 5,000$ deliveries annually. We abstracted test results, age, race, and ZIP code from electronic medical records and validated the data for all deliveries at both hospitals through July 29, 2020. Patient residential ZIP code was linked with data from the US Census Bureau to estimate median household income, proportion of households below federal poverty limit, use of public transit to travel to work, average household size, proportion of crowded households $(>1$ person per room), population density per square mile, neighborhood deprivation index, and proportion minority (nonwhite) residents (2). Data on neighborhood characteristics were derived from American Community Survey 5-year estimates for 2014-2018. Characteristics were calculated at the census tract level and linked to patient resident address using a validated ZIP code to tract crosswalk (6). To calculate the neighborhood deprivation index, we conducted principal components analysis using data for all census tracts in Georgia on 8 sociodemographic indicators, framed around domains of income, education, employment, and housing quality, producing a summary score ranging from -1.85 to 3.87 , centered at 0 ; higher values indicated higher deprivation or more disadvantage.

We examined distributions of sociodemographic (age, race/ethnicity, and insurance status) and neighborhood characteristics by delivery hospital; we then combined data from both institutions to calculate prevalence rates and 95\% CIs. For prevalence calculations, we dichotomized median income, proportion nonwhite residents, proportion below federal poverty limit, use of public transit, household crowding, neighborhood density, and neighborhood deprivation index by $<50$ th percentile and $\geq 50$ th percentile. We performed data analysis using R (https://www.r-project.org); p values $<0.05$ were considered statistically significant.

We captured data on 1,882 women after delivery who had available test results, out of a total of 2,196 deliveries (Table; Appendix Figure, https:// wwwnc.cdc.gov/EID/article/26/11/20-3091-App1. pdf). Overall SARS-COV-2 infection rate was $4.1 \%$ $(77 / 1,882)$; prevalence was higher at the safety net hospital $(9.4 \%, 58 / 616)$ than at the academic hospital $(1.5 \%, 19 / 1,266)$. The study population was predominantly non-Hispanic Black $(81.1 \%, 1,526 / 1,882)$ and publicly insured $(43.9 \%, 826 / 1,882)$.

The prevalence of SARS-CoV-2 was highest among women who were Hispanic $(15.8 \%$, 95\% CI $9.8-21.9 ; \mathrm{p}<0.001)$ or uninsured $(10.1 \%, 95 \%$ CI $5.5-$ 14.6; $\mathrm{p}<0.001)$. Prevalence was also higher for women living in census tracts with smaller average household size $(5.2 \%, 95 \%$ CI 3.8-6.6. vs. 3.0\%, 95\% CI 1.9-4.1; p $=0.02)$, increased neighborhood density $(5.1 \%, 95 \%$ CI 3.7-6.5, vs. 3.1\%, 95\% CI 2.0-4.2; p = 0.03). We observed non-statistically significant increases in prevalence in census tracts with lower average household income, increased proportion of households below the federal poverty limit, and more neighborhood deprivation. 
Table. Sociodemographic and neighborhood characteristics associated with increased prevalence of severe acute respiratory syndrome coronavirus 2 infection in laboring women, Georgia, USA*

\begin{tabular}{|c|c|c|c|c|}
\hline Demographics & No. patients, $N=1,882$ & $\begin{array}{c}\text { Total tested } \\
\text { positive }\end{array}$ & $\begin{array}{c}\text { Prevalence, \% } \\
(95 \% \mathrm{Cl}) \dagger\end{array}$ & $p$ value \\
\hline \multicolumn{5}{|l|}{ Patient } \\
\hline Age, y & & & & 0.34 \\
\hline$<20$ & 108 & 7 & $6.5(1.8-11.12)$ & \\
\hline $20-35$ & 1,393 & 57 & $4.1(3.1-5.1)$ & \\
\hline$>35$ & 381 & 13 & $3.4(1.6-5.2)$ & \\
\hline Race/ethnicity & & & & $<0.001$ \\
\hline Non-Hispanic Black & 1,353 & 52 & $3.8(2.8-4.9)$ & \\
\hline Non-Hispanic White & 226 & 0 & 0 & \\
\hline Hispanic & 139 & 22 & $15.8(9.8-21.9)$ & \\
\hline Asian/other & 164 & 3 & $1.8(-0.2$ to 3.9$)$ & \\
\hline Insurance type & & & & $<0.001$ \\
\hline Commercial & 698 & 11 & $1.6(0.7-2.5)$ & \\
\hline Medicaid/Medicare & 1,015 & 49 & $4.8(3.5-6.1)$ & \\
\hline Uninsured & 169 & 17 & $10.1(5.5-14.6)$ & \\
\hline \multicolumn{5}{|l|}{ Neighborhood $†$} \\
\hline Median household income & & & & 0.06 \\
\hline Lower average income & 934 & 47 & $5.0(3.6-6.4)$ & \\
\hline Higher average income & 939 & 30 & $3.2(2.1-4.3)$ & \\
\hline Below federal poverty limit & & & & 0.20 \\
\hline Lower poverty & 924 & 32 & $3.5(2.3-4.6)$ & \\
\hline Higher poverty & 949 & 45 & $4.7(3.4-6.1)$ & \\
\hline Use of public transit & & & & 0.25 \\
\hline Less transit use & 950 & 34 & $3.6(2.4-4.8)$ & \\
\hline More transit use & 923 & 43 & $4.7(3.3-6.0)$ & \\
\hline Proportion non-White & & & & 0.73 \\
\hline Lower percent minority & 944 & 37 & $3.9(2.7-5.2)$ & \\
\hline Greater percent minority & 929 & 40 & $4.3(3.0-5.6)$ & \\
\hline Household crowding & & & & 0.02 \\
\hline Small household size & 944 & 49 & $5.2(3.8-6.6)$ & \\
\hline Large household size & 929 & 28 & $3.0(1.9-4.1)$ & \\
\hline Neighborhood density & & & & 0.03 \\
\hline Less dense & 911 & 28 & $3.1(2.0-4.2)$ & \\
\hline More dense & 958 & 49 & $5.1(3.7-6.5)$ & \\
\hline Neighborhood deprivation index & & & & 0.64 \\
\hline Less deprived & 935 & 36 & $3.9(2.6-5.1)$ & \\
\hline More deprived & 938 & 41 & $4.4(3.1-5.7)$ & \\
\hline
\end{tabular}

This study leveraged a universal SARS-COV-2 testing program in an obstetric cohort to determine sociodemographic and neighborhood risk factors for infection. Infection was significantly associated with Hispanic ethnicity, uninsured status, high neighborhood density. The counterintuitive findings for household size may be due to chance or the narrow range of average household size across included neighborhoods (1.5-3.8 persons/household) (1).

This study has several limitations, including retrospective data collection and limited generalizability. However, its strengths include reporting of rates during a period of universal testing in an area outside of New York, and the use of census data to approximate neighborhood factors associated with increased risk for infection.

These data suggest that concentrated socioeconomic and neighborhood disadvantage, such as race/ethnicity, uninsured status, and neighborhood density, account for some of the disparate burden of COVID-19 illness in pregnant patients, a unique population at increased risk for perinatal illness (7). Research that considers specific features of neighborhood is needed to inform communitytargeted mitigation strategies if viral containment is to be achieved.

\section{Acknowledgments}

We thank Sallie Owens and Shanza Ashraf for their assistance with data validation.

\section{About the Author}

Dr. Joseph is a clinical fellow in the Division of Maternal Fetal Medicine in Department of Gynecology and Obstetrics at Emory University School of Medicine. 
Her current research investigates the impact of Social Determinants of Health needs and interventions on severe maternal morbidity and maternal mortality in an attempt to improve parity in maternal/neonatal health outcomes.

\section{References}

1. Emeruwa UN, Ona S, Shaman JL, Turitz A, Wright JD, Gyamfi-Bannerman C, et al. Associations between built environment, neighborhood socioeconomic status, and SARS-CoV-2 infection among pregnant women in New York City. JAMA. 2020;324:390-392 https:// doi.org/10.1001/ jama.2020.11370

2. United States Census Bureau. American community survey, 2012-2016. [cited 2020 May 23]. https:/ / www.census.gov/ programs-surveys/acs/

3. Moore JT, Ricaldi JN, Rose CE, Fuld J, Parise M, Kanget GJ et al. Disparities in incidence of COVID-19 among underrepresented racial/ethnic groups in counties identified as hotspots during June 5-18, 2020-22 states, February-June 2020. MMWR Morb Mortal Wkly Rep. https://www.cdc.gov/ mmwr/volumes/69/wr/mm6933e1.htm
4. Georgia Department of Public Health. COVID-19 daily status report. 2020 [cited 2020 May 23]. https:/ / dph.georgia.gov/ covid-19-daily-status-report

5. Jamieson DJ, Haddad LB. What obstetrician-gynecologists should know about population health. Obstet Gynecol.2018;131:1145-52. https://doi.org/10.1097/ AOG.0000000000002638

6. Wilson R, Din A. Understanding and enhancing the U.S. Department of Housing and Urban Development's ZIP code crosswalk files. Cityscape: J Policy Dev Res. 2018;20:277-94 [cited 2020 Sep 23]. https://www.huduser.gov/portal/ periodicals/cityscpe/vol20num2/ch16.pdf

7. Ellington S, Strid P, Tong VT, Woodworth K, Galang RR, Zambrano LD, et al. Characteristics of women of reproductive age with laboratory-confirmed SARS-CoV-2 infection by pregnancy status - United States, January 22June 7, 2020. MMWR Morb Mortal Wkly Rep. 2020;69:769-75. https://doi.org/10.15585/mmwr.mm6925a1

Address for correspondence: Naima T. Joseph, Department of Gynecology and Obstetrics, Emory University School of Medicine, 69 Jesse Hill Jr. Dr SE, 4th Fl, Glenn Building, Atlanta, GA 30303, USA; email: ntjose2@emory.edu

\section{COMMENT LETTERS}

\section{Nocardia ignorata Infection in Heart Transplant Patient}

\author{
Victoria A. Muggia, Yoram A. Puius \\ Author affiliations: Albert Einstein College of Medicine, Bronx, NY, \\ USA; Montefiore Medical Center, Bronx
}

DOI: https://doi.org/10.3201/eid2611.202756

To the Editor: We read with interest the recent description of pulmonary Nocardia ignorata infection (1). We report a similar infection in an orthotopic heart transplant recipient, which likely began as a pulmonary infection with dissemination to soft tissue, without known exposure. Risk factors included tacrolimus, steroids, older age, and posttransplant intensive care unit admission (2).

The patient was a 66-year-old African American man with a history of ischemic cardiomyopathy. After implantation of a left ventricular assist device, infectious complications included Enterococcus faecalis device infection and extended spectrum $\beta$-lactamase-producing (ESBL) Klebsiella urosepsis. The course after left ventricular assist device explantation and orthotopic heart transplant was complicated by tamponade requiring a pericardial window and an ESBL Klebsiella urinary tract infection treated with meropenem. Because of leukopenia, Pneumocystis prophylaxis was changed from trimethoprim/ sulfamethoxazole to atovaquone 2 weeks posttransplant. ESBL Klebsiella bacteremia recurred 6 weeks later, again treated with meropenem.

The patient returned 6 months posttransplant with 10 days of cough and dyspnea. Chest computed tomography demonstrated bilateral nodules with cavitation, bronchiectasis, and spiculation. We initially treated the patient with meropenem and doxycycline. Results from severe acute respiratory syndrome coronavirus 2 swab test, respiratory pathogen panel, fungal studies, and sputum culture were nondiagnostic. We obtained no additional pulmonary samples.

Due to severe left calf pain, venous duplex was performed, revealing a nonvascular mass. The patient reported no trauma, soil contact, or recent travel. The abscess was aspirated, demonstrating branching gram-positive beaded rods. The isolate was identified by a reference laboratory (Mycobacteria and Nocardia Laboratory, University of Texas Health Center at Tyler, Tyler, TX, USA) by partial $16 \mathrm{~S}$ rRNA sequencing as a $99.51 \%$ match with Nocardia ignorata, with susceptibilities identical to the isolate in Rahdar et al. (1). Brain magnetic resonance imaging results were unremarkable. The patient's respiratory status and leg pain quickly 